\title{
Acute Myocardial Injury During SARS-CoV-2 Pneumonia and Long-term Prognosis
}

J Gen Intern Med 37(4):1014-6

DOI: $10.1007 / \mathrm{s} 11606-021-07238-\mathrm{x}$

(C) Society of General Internal Medicine 2021

\section{INTRODUCTION}

SARS-CoV-2 infection is multisystemic and, after the lungs, the heart is the second most affected organ. Acute myocardial injury (AMI) can be detected in approximately $20-30 \%$ of hospitalized patients. ${ }^{1}$ The pathophysiology of AMI during COVID-19 are multifactorial and are mainly based on acute cardiac damage in the form of type 1 and type 2 myocardial infarction, stress-induced myocardial injury, and SARS-CoV2 associated myocarditis. ${ }^{2},{ }^{3}$ The presence of AMI is associated with worse short-term outcomes, ICU admission, and mortality. ${ }^{4}$

However, the significance of the troponin elevation on long-term consequences remains to be clarified. Our work aims to analyze the influence of acute myocardial injury at the time of hospitalization for SARS-CoV-2 on 1-year mortality.

\section{METHODS}

Hospitalized survivors due to SARS-CoV-2 pneumonia from the first COVID-19 wave (February 2nd to April 8th 2020) from a tertiary university hospital were prospectively followed for 1-year. AMI was defined as high-sensitivity troponin I concentrations above the 99th percentile upper reference limit (values of our laboratory $20 \mathrm{ng} / \mathrm{L}$ ). We compared the outcomes of patients who had AMI on admission versus those who did not. Bivariate analysis was performed to identify the characteristics of survivors and long-term mortality. Variables are expressed as median and IQR and comparations were made using U-Mann-Whitney test, Kaplan-Meier, or Cox proportional hazard regression. Statistical significance was considered for a value of $p<0.05$. The study was approved by our institutional review board (project number 50/2020).

Received July 31, 2021

Accepted October 19, 2021

Published online January 3, 2022
RESULTS

Among 337 hospitalized patients diagnosed with SARS-CoV2 pneumonia, there were 256 survivors $(75.9 \%) ; 21.9 \%$ met criteria for an AMI on admission. Clinical characteristics of patients with and without AMI are described in Table 1. Multivariate analysis showed that age $>65$ years (OR 6.33, 95\% CI 2.93-13.67), chronic kidney failure (OR 2.43, 95\% CI 1.05-5.62), and male sex (OR 1.92, 95\% CI 1.09-3.38) were independently associated with AMI. During the follow-up period (median 370 days (IQR 359-376)), mortality was $9.4 \%$ ( 24 cases). Two-thirds of mortality cases occurred during the first 6 months and $54.1 \%$ in the first 90 days of followup. $29.7 \%$ of patients with long-term mortality had ECG abnormalities. At 1 year, $80.4 \%$ of those with AMI survived, compared to $93.4 \%$ among those without an AMI. Long-term mortality was significantly associated with the presence of AMI at the initial episode (19.7\% AMI in survivors versus $45.8 \%$ in deceased patients); in our multivariable model, the only predictor of death was AMI at presentation (HR: 3.21, 95\% CI: 1.31-7.88). Other demographic and comorbidities did not predict death (Fig. 1).

\section{DISCUSSION}

Troponin elevation during COVID-19 can be a warning factor not only for short-term complications but also for long-term prognosis. Myocardial viral involvement is a recently studied pathophysiological mechanism. A prospective observational case-control study described that 71 days after the diagnosis of SARS-CoV-2 infection, $78 \%$ of the patients showed an abnormal cardiac MRI with lower left ventricular ejection fractions and higher left ventricle volumes. Furthermore, $60 \%$ had signs of inflammation regardless of pre-existing cardiovascular comorbidities. ${ }^{5}$ In addition to the hypoxic and inflammatory consequences on the stability of atheroma plaques, AMI could activate fibroblasts that lead to myocardial fibrosis with the consequent conduction disorders and development of sudden and severe arrhythmias and death. Pathological series have found hypertrophy, necrosis, edema, and interstitial hyperemia of cardiomyocytes. ${ }^{6}$ In a review of 662 autopsies, cardiac damage was observed as a result of preexisting damage from hypertension and coronary artery disease, with evidence of acute myocarditis, myocytes necrosis, 
Table 1 Baseline characteristics according to acute myocardial injury

\begin{tabular}{|c|c|c|c|c|}
\hline & Total 316 patients & No AMI $(226,71.5 \%)$ & AMI $(90,28.5 \%)$ & $p$ value \\
\hline \multicolumn{5}{|l|}{ Demographic characteristics } \\
\hline Male, $n(\%)$ & $170(53.8)$ & $113(50)$ & $57(63.3)$ & 0.032 \\
\hline Age $>65 \mathrm{y}, n(\%)$ & $196(62)$ & $117(51.8)$ & $79(87.8)$ & $<0.01$ \\
\hline \multicolumn{5}{|l|}{ Comorbidities* } \\
\hline \multicolumn{5}{|l|}{ Smoking habit, $n(\%)$} \\
\hline Hypertension, $n(\%)$ & $161(50.9)$ & $105(46.5)$ & $56(62.2)$ & 0.011 \\
\hline Coronary artery disease, $n(\%)$ & $26(8.2)$ & $10(4.4)$ & $16(17.8)$ & $<0.01$ \\
\hline Heart failure, $n(\%)$ & $28(8.9)$ & $14(6.2)$ & $14(15.6)$ & 0.008 \\
\hline Arrhythmia, $n(\%)$ & $29(9.2)$ & $15(6.6)$ & $14(15.6)$ & 0.013 \\
\hline Diabetes, $n(\%)$ & $80(25.3)$ & $50(22.1)$ & $30(33.3)$ & 0.039 \\
\hline COPD, $n(\%)$ & $20(6.3)$ & $11(4.9)$ & $9(10)$ & 0.091 \\
\hline Chronic kidney failure, $n(\%)$ & $34(10.8)$ & $12(5.3)$ & $22(24.4)$ & $<0.01$ \\
\hline Immunosuppression, $n(\%)$ & $12(3.8)$ & $11(4.9)$ & $1(1.1)$ & 0.115 \\
\hline Cerebrovascular disease, $n(\%)$ & $24(7.6)$ & $10(4.4)$ & $14(15.6)$ & 0.001 \\
\hline \multicolumn{5}{|l|}{ Physiological variables } \\
\hline Altered ECG, $n(\%)$ & $52(14.5)$ & $30(13.3)$ & $22(24.4)$ & 0.051 \\
\hline $\mathrm{SpO}_{2}<93 \%, n(\%)$ & $91(28.8)$ & $57(25.2)$ & $34(37.8)$ & 0.026 \\
\hline Lymphocyte $\left(10^{9} / \mathrm{L}\right)$, median (IQR) & $0.9(0.7-1.3)$ & $1.0(0.7-1.4)$ & $0.8(0.6-1.1)$ & 0.010 \\
\hline Neutrophile $\left(10^{9} / \mathrm{L}\right)$, median (IQR) & $4.8(3.5-7.9)$ & $4.4(3.2-6.7)$ & $6.6(4.1-10.4)$ & $<0.01$ \\
\hline Hematocrit $(\%)$ & $39.7(35.9-43)$ & $40(36.7-43.1)$ & $39.3(33.9-42.4)$ & 0.046 \\
\hline Platelet count $\left(10^{9} / \mathrm{L}\right)$, median (IQR) & $189(147-258)$ & $196(152-257)$ & $179(135-239)$ & 0.066 \\
\hline D-Dimer (ng/mL), median (IQR) & $379(210.8-793.8)$ & $305(200-603)$ & $707(364-1853)$ & $<0.01$ \\
\hline Ferritin $(\mu \mathrm{g} / \mathrm{L})$, median (IQR) & $474(239-876.5)$ & $453(216-842)$ & $542(277.25-943)$ & 0.185 \\
\hline C-reactive protein $(\mathrm{mg} / \mathrm{L})$, median (IQR) & $8(2.9-15.1)$ & $6.7(2.6-12.4)$ & $10.5(4.35-19.8)$ & 0.001 \\
\hline
\end{tabular}

${ }^{*}$ Comorbidities were selected for being known cardiovascular risk factors or influencing on hs-TnI values

Abbreviations: COPD, chronic obstructive pulmonary disease; ECG, electrocardiogram; IQR, interquartile range; hs-cTnI $>P^{99}$ (URL), highsensitivity troponin I concentrations above the 99th percentile upper reference limit; $\mathrm{SpO}_{2}$, oxygen saturation

epicardial lymphocytic infiltrates, lymphocytic endothelialitis, and focal interstitial lymphocytic infiltrates. ${ }^{6}$

One limitation of our study is that we have evaluated allcause mortality and not specifically cardiovascular mortality.

As shown in our results, it would be possible to identify those patients who would develop worse outcomes by measuring hs-TnI. It is reasonable to suggest that the detection of hs-TNI during the acute phase can help to select high-risk patients for strict cardiac monitoring during the convalescence phase. Our study suggests that elevated troponin at admission with COVID-19 pneumonia is a marker for mortality over the

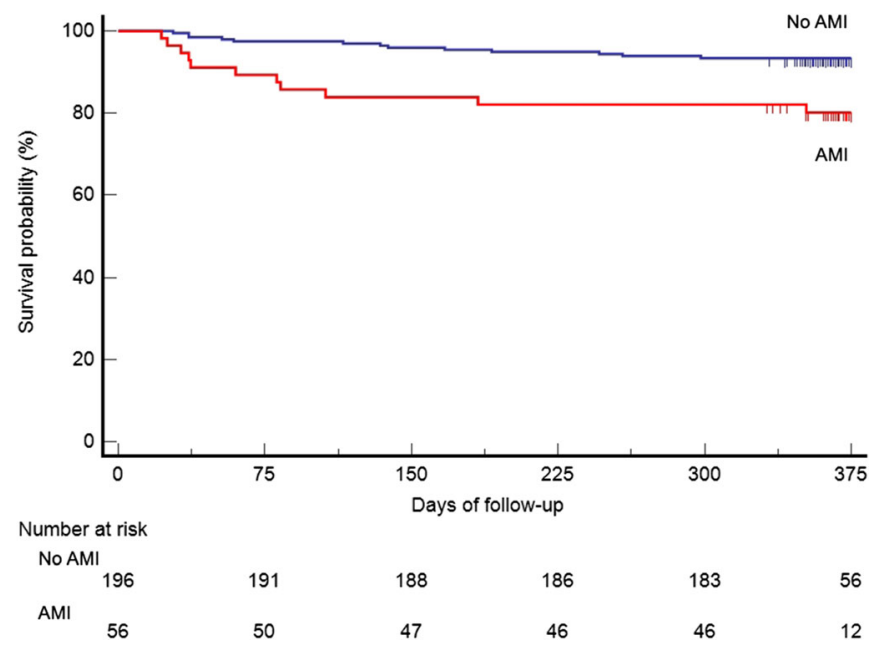

Figure 1 Survival curve of patients that survived SARS-CoV-2 pneumonia stratified by elevated AMI. subsequent year. Beyond aggressively managing cardiac risk factors, it is unclear what further steps clinicians should take.

Acknowledgements: FS, FP, JM, EF-F, JJT, and MGD contributed equal to the manuscript redaction.

Francisco Sanz, $M D, P h D^{1}$

Francesc Puchades, $M D^{2}$

Josep Melero, $M D^{3}$

Estrella Fernández-Fabrellas, $M D, P h D^{1}$

Juan José Tamarit, $M D, P h D^{2}$

Miguel García Deltoro, $\mathrm{MD}, \mathrm{PhD}^{4}$

${ }^{1}$ Pulmonology Department, Consorci Hospital General Universitari,

València, Spain

${ }^{2}$ Internal Medicine Department, Consorci Hospital General Universitari,

València, Spain

${ }^{3}$ Cardiology Department, Consorci Hospital General Universitari,

València, Spain

${ }^{4}$ Infectious Diseases Department, Consorci Hospital General Universitari,

València, Spain

Corresponding Author: Francisco Sanz, MD, PhD; Pulmonology Department, Consorci Hospital General Universitari, València, Spain (e-mail: sanz_fraher@gva.es).

\section{Declarations:}

Conflict of Interest: FS, FP, JM, EF-F, JJT, MGD declares that he/she does not have conflict of interest regarding the publication of this article. 


\section{REFERENCES}

1. Mitrani RD, Dabas N, Goldberger JJ. COVID-19 cardiac injury: Implications for long-term surveillance and outcomes in survivors. Heart Rhythm. 2020 ;17(11):1984-1990

2. Zheng YY, Ma YT, Zhang JY, Xie X. COVID-19 and the cardiovascular system. Nat Rev Cardiol. 2020;17(5):259-260

3. Wei ZY, Geng YJ, Huang J, Qian HY. Pathogenesis and management of myocardial injury in coronavirus disease 2019. Eur J Heart Fail. 2020;22(11): 1994-2006.

4. Rey JR, Caro-Codón J, Rosillo so, et al. Heart failure in COVID-19 patients: prevalence, incidence and prognostic implications. Eur J Heart Fail. 2020;22(12):2205-2215
5. Puntmann Vo, Carerj ML, Wieters I, et al. Outcomes of cardiovascular magnetic resonance imaging in patients recently recovered from coronavirus disease 2019 (COVID-19). JAMA Cardiol. 2020;5(11): 1265-1273

6. Lindner D, Fitzek A, Bräuninger $\mathbf{H}$, et al. Association of cardiac infection with SARS-CoV-2 in COVID-19 autopsy cases. JAMA Cardiol. 2020;5(11): 1281-1285

Publisher's Note: Springer Nature remains neutral with regard to jurisdictional claims in published maps and institutional affiliations. 